Proceedings of the 16th Czech and Slovak Conference on Magnetism, Košice, Slovakia, June 13-17, 2016

\title{
Novel Method of Offset Voltage Minimization in Hall-Effect Sensor
}

\author{
O. Petruk ${ }^{a}$, M. Kachniarz ${ }^{a, *}$ And R. SzeWCZyK ${ }^{b}$ \\ ${ }^{a}$ Industrial Research Institute for Automation and Measurements, Al. Jerozolimskie 202, 02-486 Warsaw, Poland \\ ${ }^{b}$ Institute of Metrology and Biomedical Engineering, Warsaw University of Technology, \\ sw. A. Boboli 8, 02-525 Warsaw, Poland
}

\begin{abstract}
The paper presents numerical model and validation of new methodology of offset voltage minimization in the Hall-effect sensors. Model of the Hall-effect sensor with multiple electric pins was developed. Mathematical equations used for calculation of electric potential difference were formulated. Simulations were carried out using finite elements method in ELMER FEM software. Performed investigation of actual parameters of newly designed Hall-effect sensor confirms effectiveness of the described method.
\end{abstract}

DOI: 10.12693/APhysPolA.131.1177

PACS/topics: 07.55.Ge, 85.75.Ss, 85.30.Fg, 02.70.Dh

\section{Introduction}

The Hall-effect sensors are the most important group within the devices for magnetic field measurements. They are utilized in many applications including DC current transformers, position detectors, incremental counters, etc. [1]. Their operation involves generation of electric potential difference (the Hall voltage) in the plate of conductor or semiconductor, when electric current is flowing to through the plate and the plate is placed in the magnetic field directed perpendicularly to the direction of current flow [2].

One of the most important problems in application of the Hall-effect sensors is occurrence of the offset voltage. It is the voltage, which can be measured in the output electrodes of the Hall-effect sensor even when external magnetic field is absent. It can be result of geometrical imperfections of the Hall-effect structure, inhomogeneities of the material or mechanical distortions [2]. Offset voltage is limiting ability of the Hall-effect sensor to detect low magnetic fields. Due to this reason, many methods of reduction of the offset voltage are under development. Most of them involves utilization of external analog circuits or resistance bridges to reduce the offset voltage $[3,4]$.

The following paper presents new concept of minimization of the offset voltage in the Hall-effect sensor, which does not requires any additional equipment. Presented methodology is validated with numerical simulation utilizing finite element method (FEM) in ELMER FEM open source software [5].

\section{Basis of the developed method}

In classical approach, Hall-effect sensor is considered as a four-electrode transducer, where between two opposite

*corresponding author; e-mail: mkachniarz@piap.pl

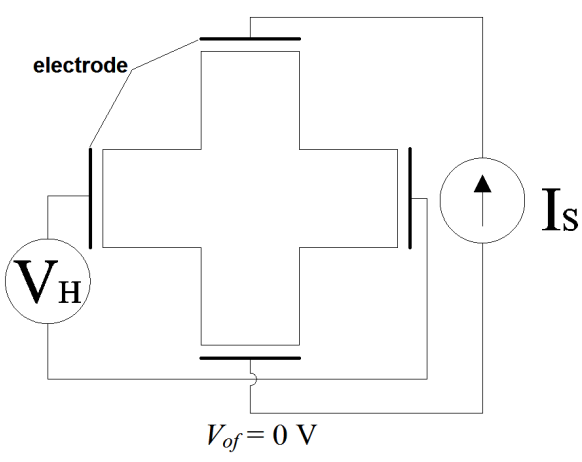

Fig. 1. Schematic representation of the Hall-effect sensor with four electrodes and without any material flaws - offset voltage $V_{o f}=0 \mathrm{~V}, I_{s}$ - supply current, $V_{H}$ - Hall voltage.

electrodes the supply current $I_{s}$ is flowing and between the other two opposite electrodes the Hall voltage is produced. It is schematically presented in Fig. 1.

In this case the perfect Hall-effect structure without any material flaws is considered, which leads to the offset voltage value $V_{o f}=0 \mathrm{~V}$.

In the real Hall-effect sensor, where the material flaws are present, the offset voltage appears to be $V_{\text {of }} \neq 0 \mathrm{~V}$. This situation is schematically presented in Fig. 2.

As the offset voltage is unnecessary and affects the measurements results, there is a need to develop methodology allowing to reduce the value of the offset voltage. The developed methodology involves replacing each of the four electrodes of the Hall-effect sensor with the parallel combination of several smaller electric pins, which can be connected in any combination (Fig. 3).

Change of the combination of connected electric pins allows to modify the resistance between each pair of adjacent electrodes and balance the voltage distribution within the Hall-effect structure, which leads to reduction of the offset voltage $\left(V_{o f} \approx 0 \mathrm{~V}\right)$. 


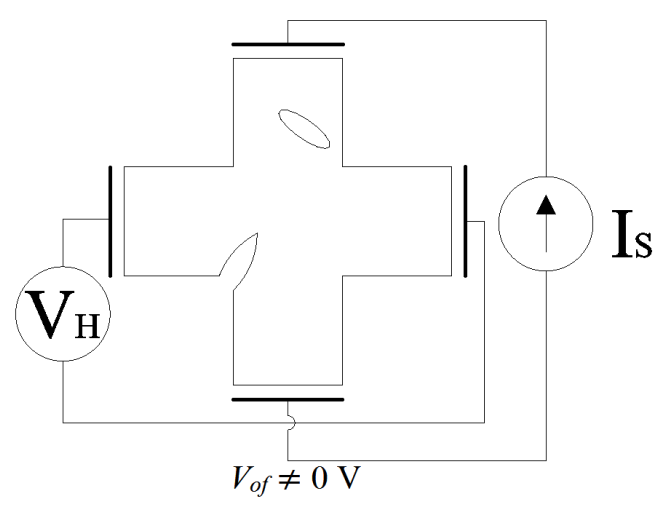

Fig. 2. Schematic representation of the Hall-effect sensor with four electrodes and the material flaws and with significant offset voltage $V_{\text {of }} \neq 0 \mathrm{~V}, I_{s}-$ supply current, $V_{H}$ - Hall voltage.

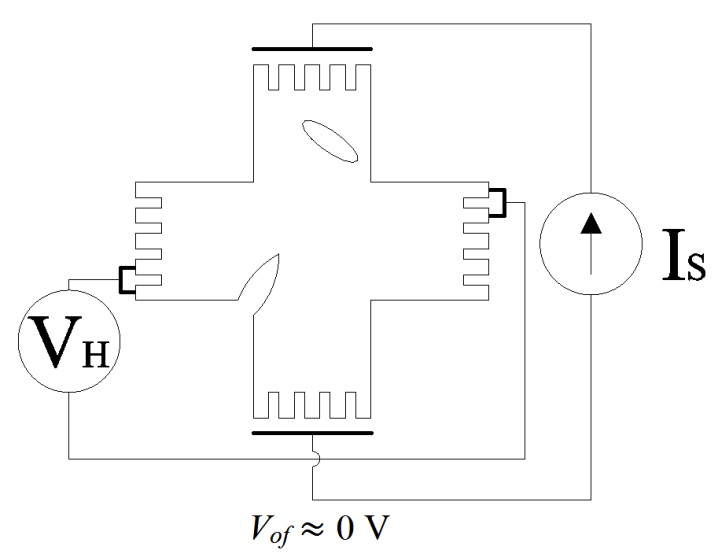

Fig. 3. Schematic representation of the Hall-effect sensor with multiple electric pins and the material flaws when only selected optimal pins are connected, offset voltage is significantly reduced $V_{o f} \approx 0 \mathrm{~V}, I_{s}$ - supply current, $V_{H}$ - Hall voltage.

\section{Simplified Maxwell equations for electric potential calculation}

Developed idea of the Hall-effect sensor with multiple electric pins was modeled using FEM in ELMER FEM software. Authors of the software developed special solver StatCurrentSolve for solving differential equations in static current conduction issues [6], which was used for modeling electric potential distribution in the Hall-effect structure. Solver is based on the electroquasistatic approximation of the Maxwell equations [6]:

$$
\begin{aligned}
& \nabla \cdot \boldsymbol{D}=\rho, \\
& \nabla \times \boldsymbol{E} \cong 0, \\
& \nabla \times \boldsymbol{H}=\boldsymbol{J}+\frac{\partial \boldsymbol{D}}{\partial t},
\end{aligned}
$$

where $\boldsymbol{D}$ is electric flux density vector, $\boldsymbol{E}$ is electric field vector, $\boldsymbol{H}$ is magnetic field vector, $\boldsymbol{J}$ is current density vector and $\rho$ is electic charge density. In this approach electric field can be expressed as

$$
\boldsymbol{E}=-\nabla \varphi,
$$

where $\phi$ is electric scalar potential field. The continuity equation for electric charges is obtained from Eq. (1) and Eq. (3):

$$
\frac{\partial \rho}{\partial t}+\nabla \cdot \boldsymbol{J}=0
$$

Relationship between current density and electric field, based on Ohm's law, takes form

$$
\boldsymbol{J}=\sigma \boldsymbol{E},
$$

where $\sigma$ is electric conductivity. Substituting Eqs. (4) and (6) to Eq. (5) results in the Poisson equation for electrostatics

$$
\nabla \cdot \sigma \nabla \varphi=\frac{\partial \rho}{\partial t}
$$

This equation is used for clculation of electric potential. The volume current density is calculated with equation

$$
\boldsymbol{J}=-\sigma \nabla \varphi .
$$

For calculation of electric potential distribution, boundary conditions are necessary, which can be either the Dirichlet or the Neumann condition. Presented mathematical description allows to calculate electric potential distribution for given geometrical shape, for example for the Hall-effect structure.

\section{FEM modeling}

For the purpose of performed simulation, geometrical model of developed Hall-effect sensor with multiple electric pins was created. As a geometric shape, symmetrical equal-arm cross was selected, which is one of the most commonly manufactured shape of the Hall-effect structures. Geometric model was meshed with tetrahedral adaptive mesh composed of 19944 elements. The model was created for the Hall-effect structure with defects in material structure and five electric pins on each bar, which is presented in Fig. 4.

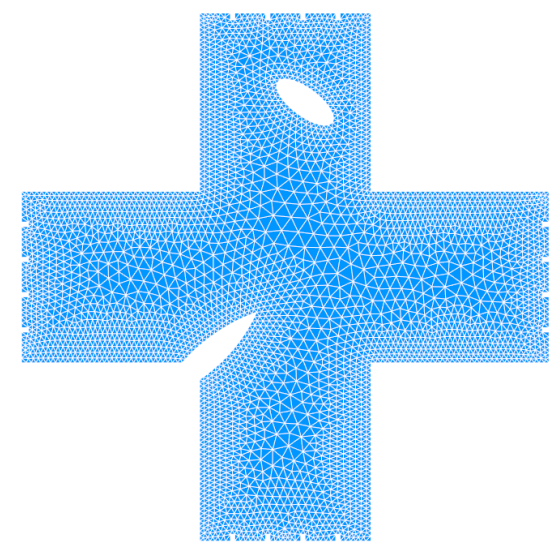

Fig. 4. Developed tetrahedral mesh for simulation of electric potential distribution in multiple-pin Hall-effect structure with material flaws.

Basing on the developed mesh, FEM simulations were performed in ELMER FEM software. Each simulation 
was performed with StatCurrentSolve solver utilizing equations described in Sect. 3 with the Dirichlet boundary conditions to solve differential equations of electric potential distribution.

First simulation was performed for the model of the Hall-effect structure with material flaws presented in Fig. 4 and all electric pins connected. The supply voltage is $1.0 \mathrm{~V}$. Result is presented in Fig. 5 .

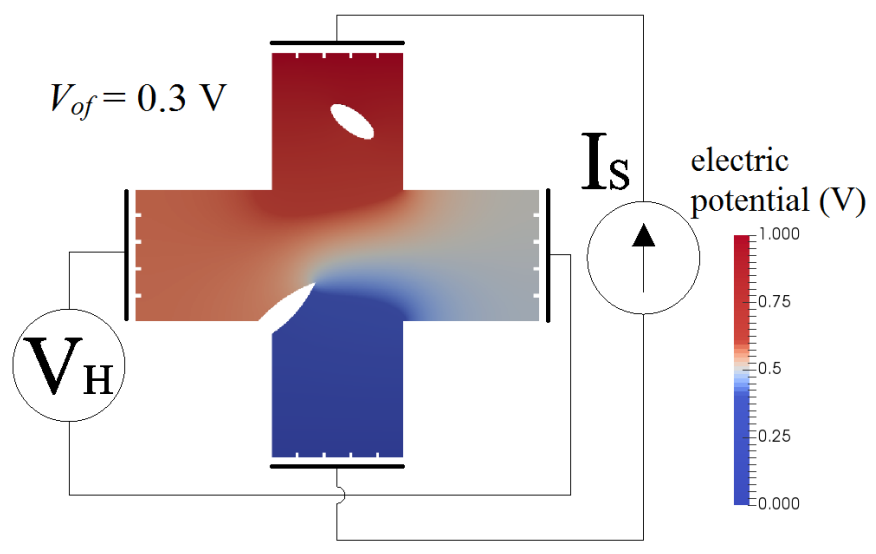

Fig. 5. Result of simulation for Hall-effect structure with material flaws and all pins connected, supply voltage $1.0 \mathrm{~V}$, offset voltage $V_{\text {off }}=0.3 \mathrm{~V}$.

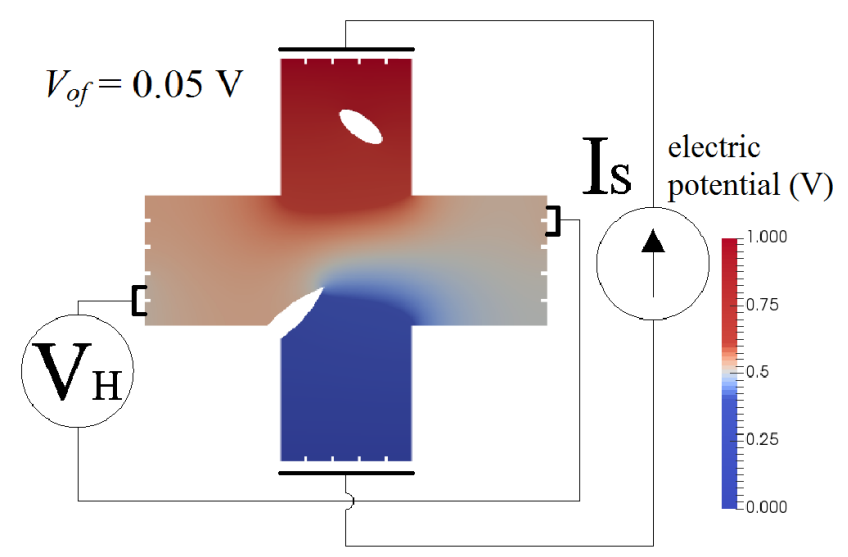

Fig. 6. Result of simulation for Hall-effect structure with material flaws and selected pins connected, connected pins in horizontal bar marked with black line, supply voltage $1.0 \mathrm{~V}$, offset voltage $V_{\text {off }}=0.05 \mathrm{~V}$.

Obtained result indicates that material flaws lead to great disturbance in the electric potential distribution in horizontal bar, where the Hall voltage is measured. This leads to significant increase of the offset voltage, which in this case is about $V_{o f}=0.3 \mathrm{~V}$. Along the vertical bar supply voltage is distributed from $0.0 \mathrm{~V}$ (ground potential) to $1.0 \mathrm{~V}$ (positive supply).

Next simulation was performed also for the structure with material flaws, but only several selected electric pins were connected in the horizontal bar to obtain optimal connection allowing to minimize value of the offset voltage. Supply voltage was again $1.0 \mathrm{~V}$. Result is shown in Fig. 6.

The result indicates significant reduction of the offset voltage (about 6 times). Selection of the proper electric pins allows to modify the resistance between electrodes of the Hall-effect structure, which leads to obtain much more uniform distribution of the electric potential in the structure with material flaws than for all electric pins connected.

\section{Conclusion}

Results of the FEM modeling presented in the paper indicates that developed methodology of minimization of the offset voltage in the Hall-effect sensors is correct and allows to obtain significant reduction of the offset voltage for the Hall-effect structure with material and geometrical imperfections. Performed modeling indicates that reduction of the offset voltage is six times.

The effectiveness of the developed method was validated on the special measurement setup and several Hall-effect structures manufactured with multiple electric pins. Measurement setup and results of the investigation were presented in the paper [7]. Reduction of the offset voltage value was up to one hundred times.

Developed methodology creates a possibility of minimization of the offset voltage during the manufacturing process, which would improve the functional parameters of the Hall-effect sensors.

\section{Acknowledgments}

This work was partially supported by the statutory founds of Institute of Metrology and Biomedical Engineering, Warsaw University of Technology (Poland).

\section{References}

[1] X. Ai, H. Bao, Y.H. Song, IEEE Trans. Power Del. 20, 11 (2005).

[2] E. Ramsden, Hall-Effect Sensor: Theory and Application, Newnes, Oxford 2006.

[3] C. Liu, J.G. Liu, in: Proc. IEEE Int. Workshop on Applied Measurements for Power System, 2014.

[4] Z.L. Warsza, J. Automat. Mobile Robot. Intellig. Syst. 4, 8 (2010).

[5] R. Szewczyk, J. Salach, J. Ruokolainen, P. Råback, K. Stefko, M. Nowicki, in: Advances in Intelligent Systems and Computing, Vol. 352, Eds. R. Szewczyk, C. Zieliński, M. Kaliczyńska, Springer, Berlin 2015, p. 219.

[6] P. Råback, M. Malinen, J. Ruokolainen, A. Pursula, T. Zwinger, Elmer Models Manual, CSC - IT Centre for Science, Espoo (Finland) 2016.

[7] M. Kachniarz, O. Petruk, R. Szewczyk, in: Advances in Intelligent Systems and Computing, Vol. 440, Eds. R. Szewczyk, C. Zieliński, M. Kaliczyńska, Springer, Berlin 2016, p. 763. 